\title{
Tratamiento de los quistes de colédoco en la edad pediátrica: Una revisión de 24 años
}

\author{
Dra. José David Palmer Becerra, ${ }^{*}$ Dra. Patricia Ulloa-Patiño
}

\section{RESUMEN}

Objetivo: Identificar la frecuencia y discutir nuestra experiencia en el tratamiento de los quistes de colédoco de niños en un periodo de 24 años.

Introducción. Los quistes de colédoco son una afección infrecuente de los conductos biliares en la edad pediátrica. Hay múltiples teorías sobre su origen. Sus manifestaciones clínicas son variables y el tratamiento es quirúrgico.

Material y métodos. Se revisaron los expedientes de 25 pacientes con diagnóstico de quiste de colédoco de agosto de 1984 a diciembre del 2008. Se registraron edad, sexo, cuadro clínico, abordaje diagnóstico, cirugía realizada, morbilidad y mortalidad.

Resultados. Hubo 18 niñas (72\%) y siete niños (28\%), la media de edad fue 5.5 años. El síntoma cardinal fue dolor abdominal en 24 $(96 \%)$; ictericia en cuatro (16\%), masa palpable en ocho (32\%); vómitos en seis $(24 \%)$. El tipo de quiste de colédoco más común fue el tipo I en la clasificación de Todani en 23 niños (92\%) y el tipo II en dos (8\%). El ultrasonido abdominal permitió el diagnóstico en el 100\% de los casos. El tratamiento quirúrgico fue la resección completa del quiste con colecistectomía y hepáticoyeyunoanastomosis en $Y$ de Roux en 18 pacientes (72\%); en siete niños (28\%) se realizó el procedimiento de Lilly con colecistectomía y hepáticoyeyunoanastomosis con $Y$ de Roux. La morbilidad fue de $12 \%$ y la mortalidad de $8 \%$.

Conclusiones. Los quistes de colédoco en niños son infrecuentes en nuestra experiencia. La variedad más frecuente fue la tipo I; el pronóstico es bueno.

Palabras clave: Quistes congénitos, colédoco, hepáticoyeyunoanastomosis, clasificación de Todani, colecistectomía.

\section{ABSTRACT}

Objective: To identify the frequency and to discuss our experience in the treatment of the choledochal cyst of children in 24 years. Introduction. Choledochal cysts are a rare entity of the bile duct in childhood. There are many theories about their origin and clinical variables. Treatment is surgical.

Material and methods. The charts of patients diagnosed with choledochal cyst were reviewed from August 1984 to December 2008 . Age, sex, clinical features, diagnostic method, surgery performed, morbidity and mortality were recorded.

Results. There were 25 patients, 18 females (72\%) and 7 males (28\%), mean age was 5.5 years. Abdominal pain the main symptom in 24 patients (96\%); jaundice in 4 patients (16\%); a palpable mass in 8 patients $(32 \%)$ and vomiting in 6 patients (24\%). Type I of choledochal cyst was the most common according to Todani in 23 patients (92\%) ant type II in two patients (8\%). Abdominal ultrasound is diagnostic of choledochal cyst in $100 \%$ of patients. Surgical treatment was complete resection of the cyst with cholecystectomy and Lilly's method was performed in 18 patients (72\%) and Lilly's method with cholecystectomy and hepatojejunostomy with Roux-in-Y in 7 patients (28\%). Morbidity was $12 \%$ and mortality, $8 \%$.

Conclusion. Choledochal cyst is a rare entity in children. Type I is the most frequent. Prognosis is good.

Key words: Congenital cyst, choledochal, hepatojejunostomy, Todani classification, cholecystectomy.

* Cirugía Pediátrica.

** Patología.

Hospital del Niño "Dr. Rodolfo Nieto Padrón" S.S.A. Villahermosa Tabasco México.

Correspondencia: Dr. José David Palmer-Becerra. Calle Coronel Gregorio Méndez Magaña \# 2832, Colonia Atasta. CP 86110. Villahermosa, Tabasco México. E-Mail: drjosepalmer@hotmail.com Recibido: agosto, 2009. Aceptado: noviembre, 2009.

Este artículo debe citarse: Palmer-Becerra JD, Ulloa-Patiño P. Tratamiento de los quistes de colédoco en la edad pediátrica: una revision de 24 años. Acta Pediatr Mex 2010;31(1):11-15.

La versión completa de este artículo también está disponible en: www.nietoeditores.com $\mathrm{mx}$

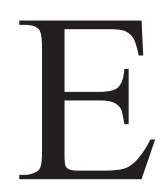

1 término quiste de colédoco ha sido aplicado a un grupo heterogéneo de entidades quísticas poco comunes de las vías biliares, se estima una frecuencia de 1 en 150,000 habitantes ${ }^{1}$. Los quistes se dividen en seis tipos según la clasificación de Alonso-Lej (1959) modificada por Todani en 1977; la dilatación quística sacular o fusiforme del colédoco es la más común ${ }^{2}$. En todos los mecanismos etiológicos propuestos existe una relación común: la obstrucción distal congénita o adquirida. La teoría del reflujo pancreatobiliar 
causado por una unión pancreatobiliar anómala es la más mencionada y estudiada. Está basada en estudios dinámicos mediante colangiopancreatografia con resonancia magnética induciendo la estimulación de la vía pancreatobiliar con secretina ${ }^{3}$. El estudio que proporciona la mayor ayuda diagnóstica es el ultrasonido abdominal, y permite observar toda la vía biliar y el páncreas.

El tratamiento de los quistes de colédoco es quirúrgico. La técnica de elección es la resección total del quiste con colecistectomía y hepáticoyeyunoanastomosis tipo Y de Roux o una cistectomía como la descrita por Lilly ${ }^{4}$. En este trabajo, comunicamos nuestra experiencia en el tratamiento de los quistes de colédoco, su tratamiento quirúrgico, la evolución clínica y las complicaciones a largo plazo.

\section{MATERIAL Y MÉTODOS}

Estudio retrospectivo, transversal, observacional y descriptivo en el Servicio de Cirugía Pediátrica del Hospital de Alta Especialidad del Niño "Dr. Rodolfo Nieto Padrón" en Villahermosa, Tabasco, México, de agosto de 1984 a diciembre del 2008. Se revisaron los expedientes de 25 pacientes operados con diagnóstico de quiste de colédoco. Se registraron la edad, género, cuadro clínico al momento de la presentación, estudios de gabinete, procedimiento quirúrgico realizado, complicaciones postoperatorias, así como la morbilidad y mortalidad. Estadísticamente se utilizaron medidas de tendencia central.

\section{RESULTADOS}

De los $25(100 \%)$ pacientes, 18 mujeres (72\%) y siete hombres (28\%). La edad iba de seis días a 11 años; media, 5.5 años. El seguimiento clínico se documentó sólo en los últimos $16(64 \%)$ pacientes por dificultades de accesibilidad a los expedientes clínicos de años anteriores a 1990 . De éstos el que tuvo menor seguimiento postoperatorio fue de 11 meses y el de mayor seguimiento, 48 meses. El tipo de quiste de colédoco más común fue el tipo I en 23 pacientes (92\%); el tipo II se presentó en uno (4\%) y el tipo IV en uno (4\%). El síntoma cardinal fue el dolor abdominal, en 24 pacientes (96\%); ictericia en cuatro (16\%); masa palpable en ocho (32\%); vómitos en seis pacientes (24\%). Los estudios realizados fueron: una placa simple de abdomen, radiografía de tórax y ultrasonido abdominal en 25 pacientes $(100 \%)$; tomografía computarizada con doble contraste en 11 (44\%); colangiopancreatografia retrógrada endoscópica en dos $(8 \%)$ y colangiorresonancia magnética en dos pacientes (8\%). Las patologías asociadas fueron litiasis vesicular en dos y pancreatitis en nueve. La técnica quirúrgica realizada fue la resección completa del quiste con colecistectomía y hepáticoyeyunoanastomosis tipo Y de Roux en 18 pacientes (72\%) y procedimiento de Lilly con hepáticoyeyunoanastomosis tipo Y de Roux en 6 pacientes (24\%). En el postoperatorio se administraron ampicilina y amikacina por $48 \mathrm{~h}$ en 24 pacientes (96\%). La sonda nasogástrica se dejó en los pacientes por $24 \mathrm{~h}$, se inició alimentación enteral a las $48 \mathrm{~h}$ postoperatorias. Se colocó un drenaje Penrose en el lecho quirúrgico, que se retiró a las $48 \mathrm{~h}$. Los pacientes recibieron sulfametoxazol ( $2 \mathrm{mg} / \mathrm{kg} /$ día en base a trimetoprim), por tres meses después de la cirugía. El ácido urodesoxicólico se usó en 20 pacientes $(80 \%)$. Se dieron vitaminas A, D, E, K a los 24 pacientes $(96 \%)$.

Un paciente tuvo colangitis, alteración de las bilirrubinas totales, TGO Y TGP a los 12 meses y a los 24 meses tuvo cirrosis hepática. Otro paciente tuvo anemia, trombocitopenia, tiempo de coagulación prolongado y pruebas de función hepática alteradas lo que sugirió que sufría insuficiencia hepática y actualmente está en un protocolo de trasplante hepático. El diagnóstico en un paciente (4\%) se hizo en la autopsia, ya que no se operó por haber tenido una evolución clínica desfavorable y falleció. Las complicaciones postoperatorias: desequilibrio hidroelectrolítico por hiponatremia en tres pacientes (12\%) que se corrigió con la administración de concentrado de sodio intravenoso; neumonía en un paciente (4\%), sin insuficiencia respiratoria; recibió aerosoles y cefotaxima. Tuvieron sepsis dos (8\%) a quienes se trató con meropenem. Un paciente tuvo una fístula biliar (4\%) que remitió al tratamiento con ayuno, antibióticos, sonda nasogástrica, octreotide y alimentación parenteral por siete días. Hubo dos defunciones (8\%) por sepsis, una de ellas en un lactante de diez meses de edad cuya autopsia mostró un quiste gigante del colédoco de $20 \mathrm{~cm}$ de diámetro, mayor que el hígado. La media de los días de estancia fue de 14 días (con un periodo de siete a 21 días). Un paciente (4\%) tuvo colangitis a los 12 meses y cirrosis hepática a los 24 meses de operado. De los 23 pacientes vivos (92\%), uno (4\%) sufrió insuficiencia hepática; está en espera de trasplante hepático. Los otros 22 pacientes (88\%) han tenido un seguimiento anual en la consulta externa; han tenido buena evolución. 


\section{DISCUSION}

Los quistes congénitos de colédoco son infrecuentes. Más del $60 \%$ se ve en la edad pediátrica; afectan más a las mujeres como en nuestro estudio en relación de 3.5:1 y como lo descrito en la literatura, 3 a 4:1 ${ }^{5}$. La presentación más frecuente es la dilatación sacular o fusiforme que corresponde al tipo I de la clasificación de Todani, (92\% de nuestros casos). El tamaño del quiste va desde $2.5 \mathrm{~cm}$ hasta $20 \mathrm{~cm}$, en cuyo caso ocupa gran parte de la cavidad abdominal. Los quistes gigantes pueden causar obstrucción intestinal y abdomen agudo o incluso llegar a romperse y producir una peritonitis severa. En nuestra serie sólo hubo un caso (Figura 1). Las paredes de los quistes varían en espesor desde $2 \mathrm{~mm}$ hasta $1 \mathrm{~cm}$ y están compuestos por tejido fibroso, inflamatorio y muestran necrosis ocasional (Figura 2). Se han descrito pacientes pediátricos con obstrucción del conducto biliar por cálculos de ácido cálcico y aumento de los ácidos grasos en un quiste de colédoco ${ }^{6}$. La litiasis vesicular en los quistes de colédoco se ha atribuido a una estenosis congénita del conducto hepático ${ }^{7}$.

En nuestros casos, el diagnóstico se basó en los hallazgos clínicos, de laboratorio y los estudios de imagen, entre los cuales el ultrasonido abdominal permitió visualizar los quistes de colédoco en el 100\% de los casos. La tomografía computarizada simple y la contrastada también permiten ver los quistes de colédoco y además orientan sobre la presencia de pancreatitis asociada (Figura 3). La colangiorresonancia magnética permite visualizar con mayor exactitud anatómica la vía biliar (Figura 4). La colangiopancreatografía retrógrada endoscópica (CPRE) define con mayor detalle la dilatación quística del conducto colédoco, la presencia de quistes intrahepáticos, los cambios anatómicos distales obstructivos o anómalos y la presencia de lodo biliar o litiasis vesicular.

La CPRE tiene el inconveniente de que está contraindicada en los pacientes con pancreatitis ya que aumenta el estado inflamatorio del páncreas ${ }^{8}$. La tomografía computarizada sólo fue útil en casos de pancreatitis asociada.

El tratamiento fue quirúrgico en 24 de nuestros casos (96\%). Se efectuó la resección completa del quiste con hepáticoyeyunoanastomosis tipo Y de Roux y colecistectomía en todos los casos. El procedimiento de Lilly es una opción quirúrgica cuando los quistes son grandes y están adheridos por la parte posterior de su pared a la vena porta

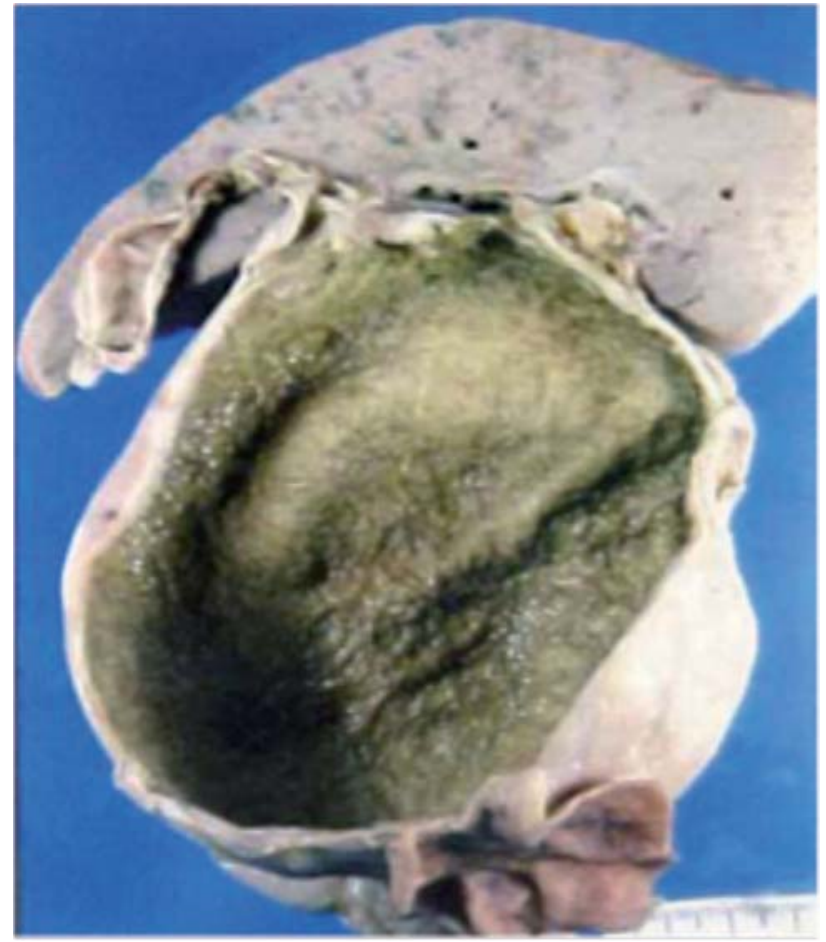

Figura 1. Quiste congénito de colédoco tipo IV en un lactante de diez meses de edad, que falleció por sepsis.

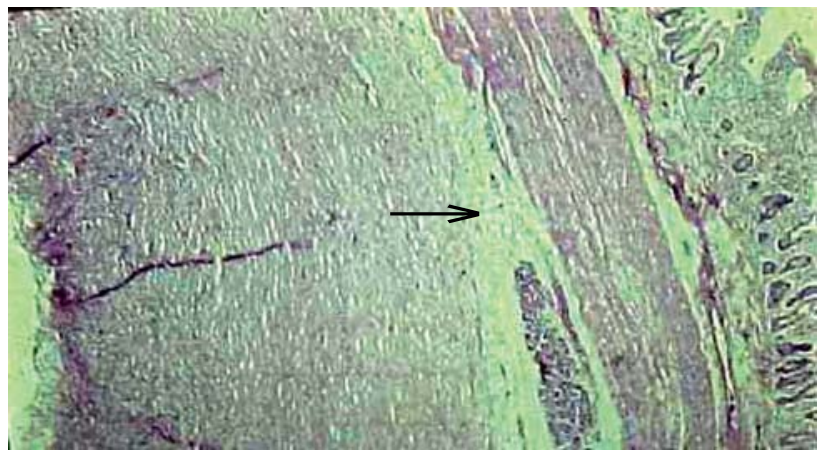

Figura 2. Microfotografía del quiste. Muestra las paredes gruesas y fibrosas con epitelio de revestimiento ulcerado. Rechaza al intestino. Se observa una porción de páncreas comprimida entre ambas estructuras.

y a la arteria hepática. El abordaje quirúrgico laparoscópico ha tenido éxito en niños entre seis meses y 12 años de edad. Tiene la ventaja de permitir una visualización y exploración exacta del hilio hepático y de poder realizar una hepáticoyeyunoanastomosis segura y efectiva ${ }^{9}$. En neonatos hay muy pocos estudios en un pequeño número de pacientes para decidir si es la mejor vía quirúrgica, aunque se han descrito resultados favorables ${ }^{10}$. 


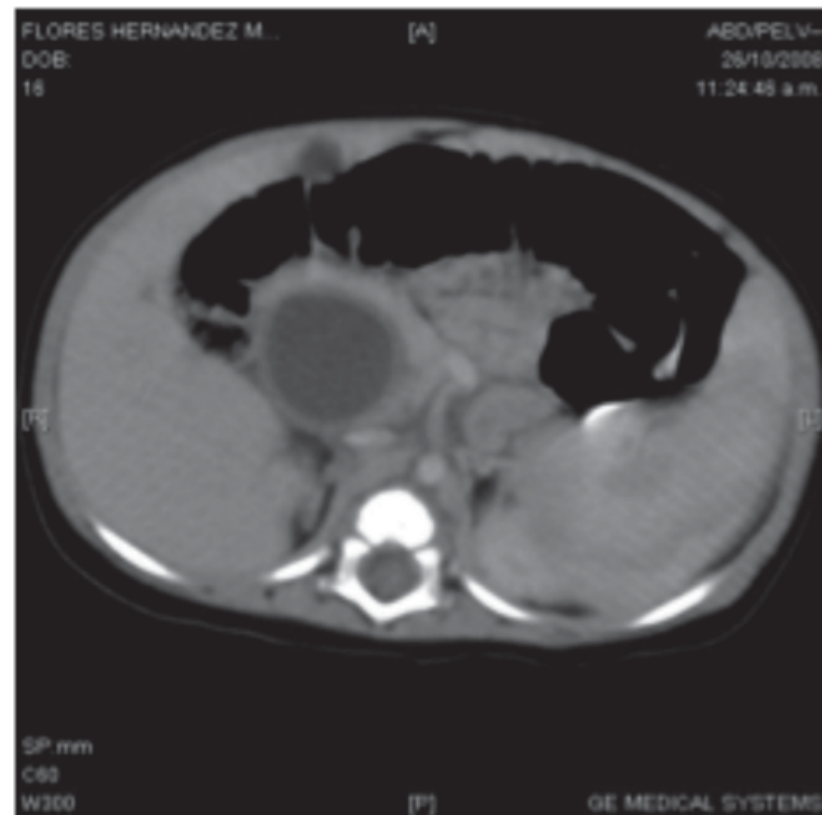

Figura 3. Tomografía computarizada de abdomen con medio de contraste. Muestra una imagen redonda de aspecto quístico con paredes finas, regulares, de contornos precisos e hipodensa a nivel de la cabeza del páncreas.

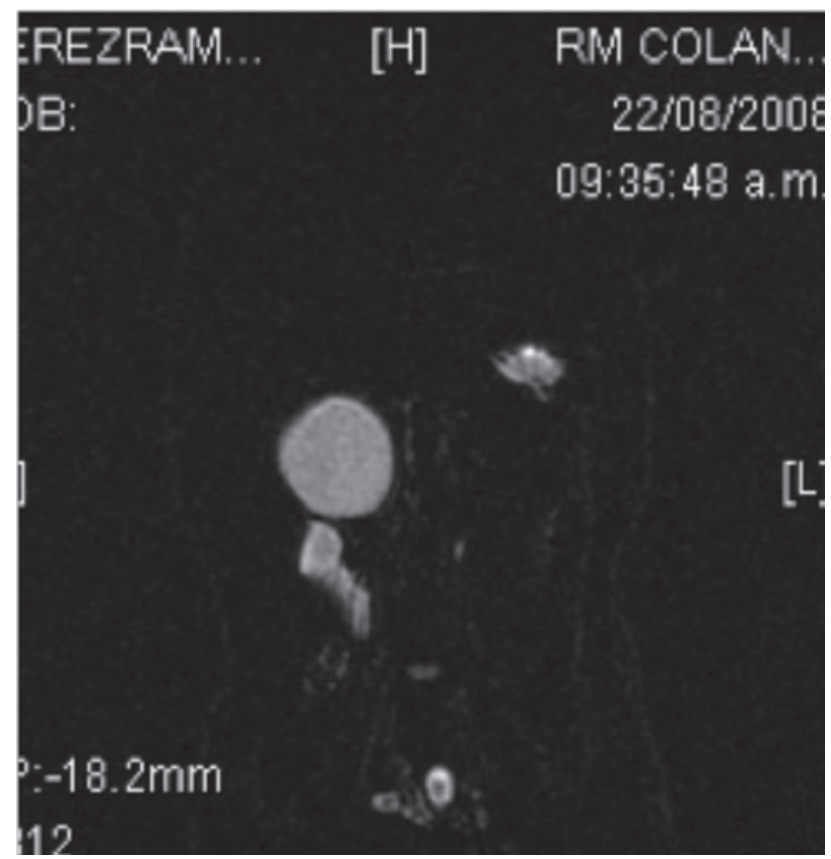

Figura 4. Colangiorresonancia magnética que muestra gran diatación quística del conducto colédoco.

Las patologías asociadas en nuestra serie fueron pancreatitis en el $36 \%$ y litiasis vesicular en $8 \%$ de los casos, cifras mayores a otros estudios nacionales ${ }^{11}$. De 17 pacientes estudiados por Orozco y cols. cinco tuvieron pancreatitis sin litiasis vesicular ${ }^{11}$. Las complicaciones de mayor gravedad son la malignización de los conductos biliares ${ }^{12}$, la estenosis de la hepáticoyeyunoanastomosis, el reflujo de la anastomosis biliodigestiva, la colangitis, cirrosis biliar, hipertensión portal e insuficiencia hepática. En nuestra serie sólo un paciente (4\%) tuvo colangitis y cirrosis hepática y dos $(8 \%)$ evolucionaron con sepsis abdominal y fallecieron.

El pronóstico de los quistes de colédoco es bueno cuando han sido operados. El seguimiento es de suma importancia, siendo necesaria la revisión mensual los tres primeros meses del postoperatorio y posteriormente cada tres meses por dos años para detectar la presencia de colangitis, en cuyo caso, la primera complicación a considerar es una estenosis de la hepáticoyeyunoanastomosis y su tratamiento debe ser con esteroides, coleréticos y en caso de persistir puede requerirse una segunda intervención para rehacer la anastomosis biliodigestiva. Sin embargo, la colangitis es una complicación rara que ocurre en menos del $5 \%$ del total de los casos.

\section{BIBLIOGRAFIA}

1. Sugiyama M, Atomi $\mathrm{Y}$, Kuroda A. Pancreatic disorders associated with anomalous pancreatobiliary junction. Surgery 2002;126:492-7

2. Todani T, Watanabe $\mathrm{Y}$, Narusue $\mathrm{M}$, et al. Congenital bile duct cyst: Classification, operative procedures, and review of 37 cases including cáncer arising from choledochal cyst. Am J Surg. 1977;134:263-9.

3. Matsufuji H, Araki Y, Nakamura A, Ohigashi S, Watanabe F. Dynamic study of pancreaticobiliary reflux using secretinstimulated magnetic resonance cholangiopancreatography in patients with choledochal cysts. J Pediatr Surg. 2006;41:1652-

4. Lilly JR. Total excision of choledochal cyst. Surg Gynecol Obstet. 1978;146:254-8.

5. Siuna K, Tsuo T. Symptoms in choledocal cyst, age-related incidence. Arch Surg. 2006;118:783-7.

6. Kaneko K, Ono Y, Tainaka T, Sumida W, Ando H. Fatty acid calcium stones in patients with pancreatobiliary maljunction/ choledochal cyst as another cause of obstructive symptoms besides protein plugs. J Pediatr Surg. 2008;43:564-7.

7. Kemmotsu H, Mouri T, Muraji T. Congenital stenosis of the hepatic duct at the porta hepatis in children with choledochal cyst. J Pediatr Surg. 2009;44:512-6.

8. Palier T, Jalets M, Marrion G. Images in choledochal cyst: Review of world literature. Hepatogastroenterology. 2008;76:398402.

9. Li L, Liu S, Hou W, Cui L, Liu X, Jun Z, Ming H, Gang L, Kamal 
N. Laparoscopic correction of biliary duct stenosis in choledocal cyst. J Pediatr Surg. 2008;43:644-6.

10. Li S, Long L, et al. Laparoscopic excision of choledochal cyst and Roux-in- $Y$ hepaticojejunostomy in symptomatic neonates. J Pediatr Surg. 2009;44:508-11.

11. Orozco SJ, Sámano MA. Dilatación quística congénita del colédoco en pediatría. Experiencia en el hospital Juárez de México. Cir Ciruj. 1997;65:33-8.

12. Ono S, Aakai K, Kimura O, Iwai N. Development of bile duct cancer in a 26-year-old man after resection of infantile choledochal cyst. J Pediatr Surg. 2008;43:E17-E19.

Consulte Acta Pediátrica de México en internet:

\author{
www.imbiomed.com.mx \\ www.actapediatrmex.entornomedico.org \\ www.intramed.net \\ www.nietoeditores.com.mx \\ www.artemisa.org.mx \\ E-mail: \\ actapediatrmex@entornomedico.org
}

